\title{
Increased toxicities in children with Burkitt lymphoma treated with Rituximab - Experience from a tertiary cancer centre In India
}

\author{
Shyam Srinivasan ${ }^{1}$, Nirmalya Roy Moulik ${ }^{1}$, Anand KC ${ }^{1}$, Gaurav Narula ${ }^{1}$, Maya Prasad ${ }^{2}$, \\ Chetan Dhamne ${ }^{2}$, BADIRA CHERIYALINKAL PARAMBIL ${ }^{2}$, Sneha Shah ${ }^{2}$, Tanuja \\ Sheth $^{1}$, Sumeet Gujral ${ }^{1}$, and Sripad Banavali ${ }^{2}$ \\ ${ }^{1}$ Tata Memorial Centre \\ ${ }^{2}$ Tata Memorial Hospital
}

May 29, 2020

\begin{abstract}
Background: Even though rituximab has emerged as the standard of care for management of high risk paediatric burkitt lymphoma(BL) its safety in children from the low-middle income countries(LMICs) remains to be proven. We herein report our experience of using rituximab in patients with BL treated in our institute. Patients and Methods: All patients diagnosed of BL between January-2015 through December-2017 were treated in a risk stratified manner with either modified MCP-842 or modified LMB protocol. Patients with poor response to MCP 842 were shifted to LMB-salvage regimen. In addition, rituximab was given for selected patients of LMB group B or C. Result: Forty-two(49.4\%) of 85 analyzed patients with BL received rituximab [Median dose:1500(Range:375-1875) mg/m2]. The incidence of febrile neutropenia $(\mathrm{p}=0.02)$, pneumonia $(\mathrm{p}=0.005)$, Intensive care unit admissions $(\mathrm{p}=0.002)$ and toxic deaths $(\mathrm{p}=0.04)$ were higher amongst BL patients who received rituximab. Pneumonia was fatal in 11 of $16(69 \%)$ patients who received rituximab. The mortality was $100 \%$ for patients who developed recurrent pneumonia after completion of treatment. On multivariate analysis, rituximab continued to be significantly associated with toxic deaths, HR:11.45(95\%CI: $1.87-70.07 ; \mathrm{p}=0.008)$. The addition of rituximab to intensive chemotherapy resulted in an inferior 1-year event free survival $(49.4 \pm 8.1 \%$ vs $79.3 \pm 6.5 \% ; \mathrm{p}=0.025)$ and 1 -year overall survival $(63.1 \pm 8.5 \%$ vs $91.8 \pm 4.5 \% ; \mathrm{p}=0.007)$. Also, the addition of rituximab did not improve 1-year relapse free survival ( $78.3 \pm 7.3 \%$ vs $83.9 \pm 6.0 \%$; $=0.817)$. Conclusion: The potential immunomodulatory effect of rituximab and increased susceptibility to infections in patients from LMICs being treated under resource-constrained situations has to be carefully considered while choosing this drug in the treatment BL.
\end{abstract}

\section{Hosted file}

Main document.docx available at https://authorea.com/users/327643/articles/455259-increasedtoxicities-in-children-with-burkitt-lymphoma-treated-with-rituximab-experience-from-atertiary-cancer-centre-in-india

\section{Hosted file}

Tables.docx available at https://authorea.com/users/327643/articles/455259-increasedtoxicities-in-children-with-burkitt-lymphoma-treated-with-rituximab-experience-froma-tertiary-cancer-centre-in-india 
Figure 1: Flow diagram with treatment, response assessment and outcome in patients with Burkitt lymphoma

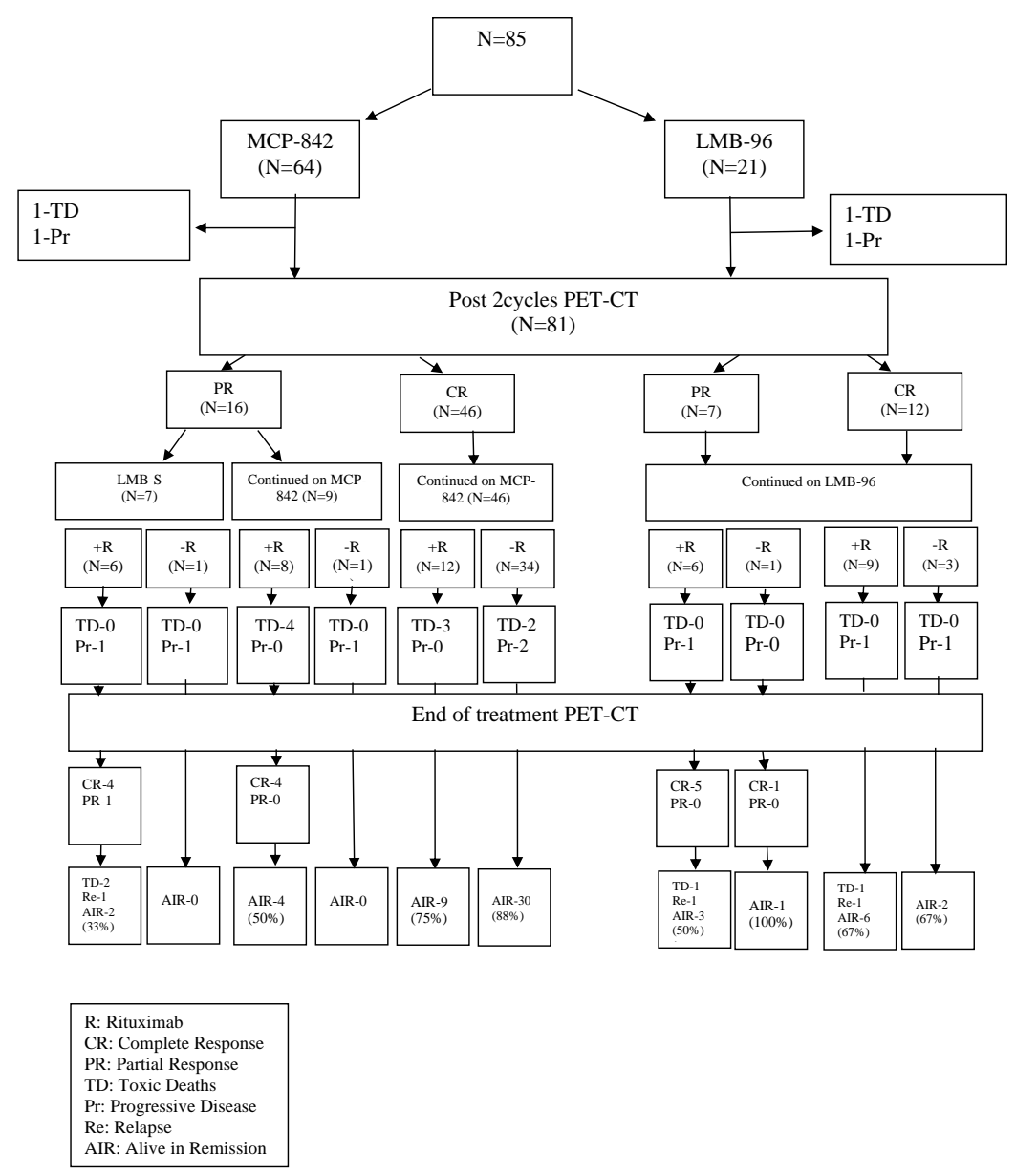

